\title{
THE NATURE OF COMPACT RADIO CORES IN GALAXIES
}

\author{
H. FALCKE
}

Max-Planck-Institut für Radioastronomie, Auf dem Hügel 69, D-53121 Bonn, Germany (hfalcke@mpifr-bonn.mpg.de

\begin{abstract}
Compact radio cores, which are often assumed to mark the presence of a super-massive black hole, are not only found in the nuclei of powerful quasars but also in nearby galaxies. While in quasars they are typically associated with relativistic jets, the nature of those cores in lowluminosity AGN is less clear. Here, I will briefly mention some of the recent theories (ADAFs or jets) and observations of the latter class of objects.
\end{abstract}

\section{Introduction}

It is often said that VLBI radio cores indicate the presence of a black hole in a galactic nucleus-but why? Three answers can be given easily: a) Because Martin Rees has said so (e.g. Lynden-Bell \& Rees 1971, where they predicted Sgr A* and achieved something few theorists ever will-they were right), $b$ ) What else should they be? (radio cores are unique objects in galactic nuclei, where super-massive black holes are though to exist), and c) because it makes a good press release (and justifies the amount of money spent on the necessary expansion of VLBI). Of course we also would like to know whether there are any other scientific arguments that prove the link between compact radio cores and black holes.

\section{Compact Radio Cores in the Galaxy and Galaxies}

In a large survey Wrobel \& Heeschen (1991) discovered that a number of elliptical galaxies have compact radio nuclei. Fabian \& Rees (1995) interpret these as the emission from a hot ion torus. In a survey of optically selected low-luminosity AGN (here only LINERs; see also Ho 1997) Falcke et al. (1997a) find that a quarter of nearby LINER galaxies have compact, flat-spectrum radio cores. Comparison of radio and $\mathrm{H} \alpha$ flux shows that in 
LINERs with steep-spectrum radio emission, radio and optical flux are not correlated, while for LINERs with flat-spectrum cores there is a marked trend for the radio flux to increase with the emission-line flux. This is a clear sign that optical and radio activity in those low-luminosity LINERs have a common origin-most likely an AGN.

Narayan (e.g. 1997) - conceptually not very different from Fabian \& Rees-suggests that those radio cores could be explained by an advection dominated accretion flow (ADAF). However, the radio spectral indices we find in LINERs are usually substantially flatter than those predicted in the ADAF model, and VLBI of some of those cores (e.g. NGC 4258, Herrnstein 1997) have revealed jet structures similar to those in more luminous AGN. Herrnstein, with his superb data, now also puts strong upper limits on any advection dominated disk in NGC 4258 . On the other hand, the newly discovered radio jet in NGC 4258 fits in many details very well the predictions of the jet-disk model by Falcke (1996), as does the $\mathrm{H} \alpha$ /radio correlation for the LINER sample (Falcke et al. 1997a).

Hence, despite the overwhelming momentum of the ADAF band wagon, I am very confident, that most, if not all, of the more prominent radio cores in nearby galaxies will turn out to be just scaled down AGN jets-thus making the black hole/compact radio core link a rather indirect one (there is a black hole, which accretes matter, which then leads to the formation of a jet, and which then looks like a compact radio core).

Only for the closest radio core, $\mathrm{Sgr} \mathrm{A}^{*}$, which hides behind a scattering screen, the game is still wide open-but there is hope. In a recent campaign to measure the simultaneous spectrum of Sgr A* (Falcke et al. 1997b) we were able to confirm the excess in its spectrum at $\mathrm{mm}$-wavelengths. This is best interpreted as an ultra-compact emission region, very close to the event horizon of the black hole. This region could then in principle be resolved already with the next generation of mm-VLBI experiments, which should have a beam smaller than the expected 'visual' size of the black hole, and thus would not only discriminate between the various models but could also finally prove the existence of a black hole.

\section{References}

Fabian, A.C., \& Rees, M.J. 1995, MNRAS 277, L55

Falcke, H. 1996, ApJ 464, L67

Falcke, H., Ho, L.C., Wilson, A.S. 1997a, in "Relativistic Jets in AGN", Cracow 1997, Ostrowski et al. (eds.)

Falcke, H., Goss, W.M., Matsuo, H. et al. 1997b, ApJ Lett., subm.

Herrnstein, J. 1997, this volume

Ho, L.C. 1997, this volume

Lynden-Bell, D., \& Rees, M.J. 1971, MNRAS 152, 461

Narayan, R. 1997, this volume

Wrobel, J.M., \& Heeschen, D.S. 1991, AJ 101, 148 